\title{
"Asked to See Patient" (ATSP)—Improving Patient Safety and Confidence in Junior Doctors
}

\author{
Shazia Hafiz, Hayley Mcmanus \\ Health Education North West, Manchester, UK
}

\begin{abstract}
"Asked to See Patient" (ATSP) is a peer-delivered teaching programme delivered to new Foundation Year One (FY1) doctors in North West England prior to the August change over period. The ATSP teaching is based on a booklet containing guidance on the management of common clinical scenarios encountered during out of hours shifts "on-call", to aid the transition from medical student to newly qualified doctor. In June every year, an ATSP "Teach the Teachers" course is delivered by the ATSP chairs. This is a one-day course delivered to existing FY1 doctors from all of the National Health Service (NHS) trusts in North West England. ATSP is subsequently delivered by the existing FY1 doctors locally at each trust in small groups. Role-play and PowerPoint presentations are used to deliver these sessions and ATSP booklets are handed out at the end. New FY1 doctors who attended the ATSP programme were then surveyed one month after starting work to assess the value of the ATSP course/booklet during on-call shifts. This electronic survey contained qualitative and quantitative assessments and these data were then collated and analysed using both statistical and thematic analysis. There were 548 FY1 doctors attended the ATSP course at their hospitals. Of the 141 who responded to the questionnaire, 58\% utilised ATSP booklets during on-calls, $59 \%$ reported improvements in prioritisation of on-call jobs, and $76 \%$ felt that they had become a safer clinician as a result of ATSP. The ATSP programme has shown to aid the transition from medical student to confident newly qualified FY1 doctor, who can initiate assessment, investigation, and management of patients prior to obtaining senior help.
\end{abstract}

Keywords: peer-delivered teaching, prioritisation, patient safety, out of hours, assessing acutely unwell patients, documentation

\section{Introduction}

The transition from medical student to newly qualified doctor is understandably daunting. One of the most anxiety-provoking aspects of this transition is prospect of working out of hours "on-call" shifts with the requirement to function as an independent clinician and treat acutely unwell patients (Brennan et al., 2010). Despite acquiring the necessary knowledge at university, one of the difficulties new doctors face is the application of this knowledge under pressure.

Peer-assisted learning is an effective method of teaching and has shown to be well received by both teachers and students (Glynn, MacFarlane, Kelly, Cantillon, \& Murphy, 2006). Being taught by teachers who understand first-hand the challenges faced by their students is one of the factors which makes it so effective. 
"Asked to See Patient" (ATSP) is a peer-delivered teaching programme delivered to newly qualified Foundation Year One (FY1) doctors in the North Western Foundation School, prior to commencing their new jobs. It was set up to ease the transition from medical student to doctor and aid the application of knowledge gained at university to on-call scenarios. The aim of the programme was not to teach new knowledge, but instead to enable the new doctors to apply their existing knowledge in a systematic, rational and ultimately, safe manner. The intention was to produce calm, organised junior doctors who had the confidence to initiate the first steps of treatment in an acute situation, prior to seeking senior advice (Dornan, Boshuizen, King, \& Scherpbier, 2007).

The programme has been delivered in the North Western Foundation School since 2011. Each year, a group of current FY1 doctors attend a "Teach the Teachers" training day, which equips them with the skills to deliver a teaching session to the new FY1 doctors starting at the hospital where they work. All foundation trusts provide a mandatory induction for their new foundation doctors for 5-10 working days prior to the changeover day in August. The small group peer-delivered teaching sessions take place during this induction period, an opportune time to equip the new trainees with skills and confidence, just before they start work in August. The content of the teaching is dedicated to the most common acute scenarios that a new FY1 doctor is likely to encounter when asked to see a patient on-call. The teaching sessions are interactive and involve role-play scenarios, which focus on the telephone conversation in response to a "bleep" from a nurse requesting a patient review and the initiation of a preliminary management plan at the patient bedside. A small booklet containing the cases discussed has also been produced to complement the programme. Each new FY1 doctor receives a printed copy to carry with them during their on-call shifts as a reminder (see Figures 1, 2, and 3).

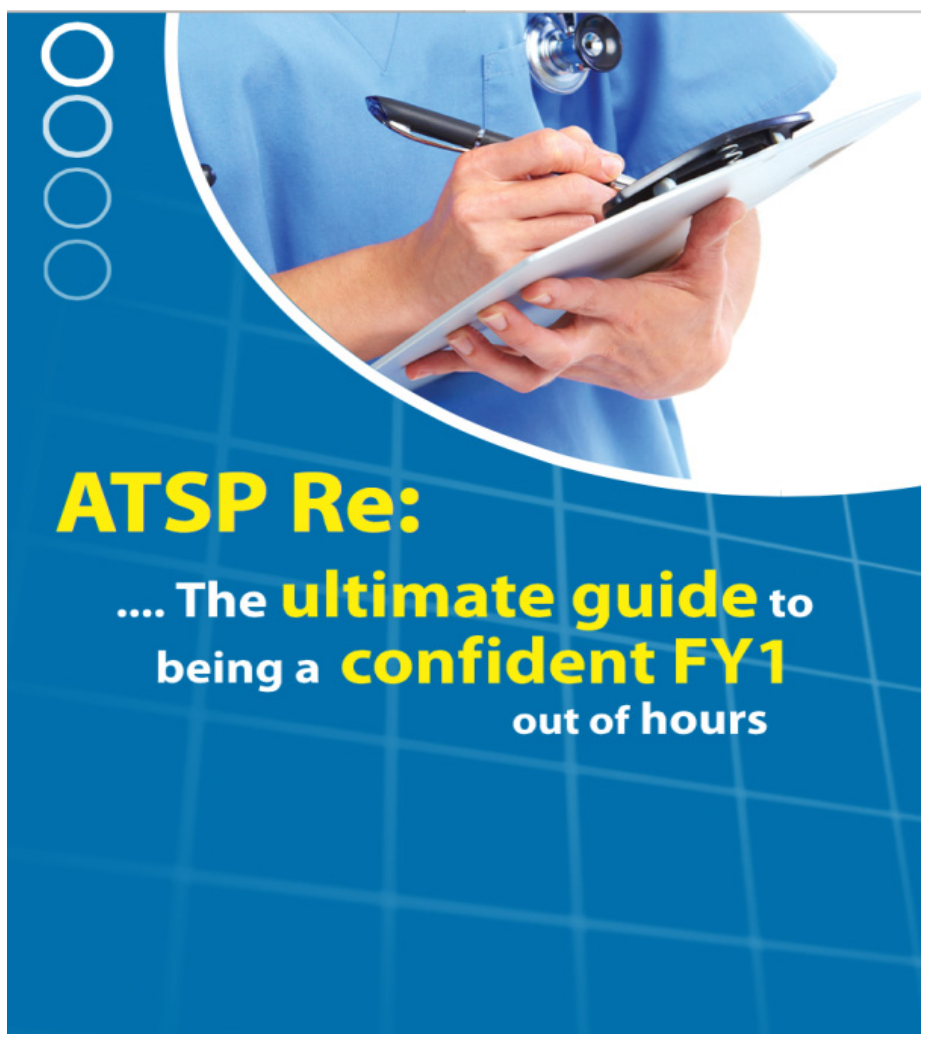

Figure 1. Front cover of the ATSP booklet. 


\section{Contents}

Page 2:

Page 3:

Page 4:

Page 5:

Page 6:

Page 7:

Page 8:

Page 9:

Page 10:

Page 11:

Page 12:

Page 13:

Page 14:

Page 15:

Page 16:

Page 18:

Page 20:
Introduction

ABDOMINAL PAIN

AGITATION/CONFUSION

BLOOD IN CATHETER BAG - Example of documentation

DECREASED GCS

DYING PATIENT

FALLS/COLLAPSE

FLUID REVIEW \& INSULIN SLIDING SCALE

HAEMATEMESIS/COFFEE GROUND VOMIT/MALAENA

HIGH EWS (General Assessment)

HYPERKALAEMIA (stable patient)

LOW URINE OUTPUT (catheterised patient)

SHORTNESS OF BREATH

TACHYCARDIA/PALPITATIONS

COMMONLY PRESCRIBED DRUGS

PRESCRIBING OUT OF HOURS

General Hints and Tips for seeing patients out of hours

1

Figure 2. Contents page of the ATSP booklet. 


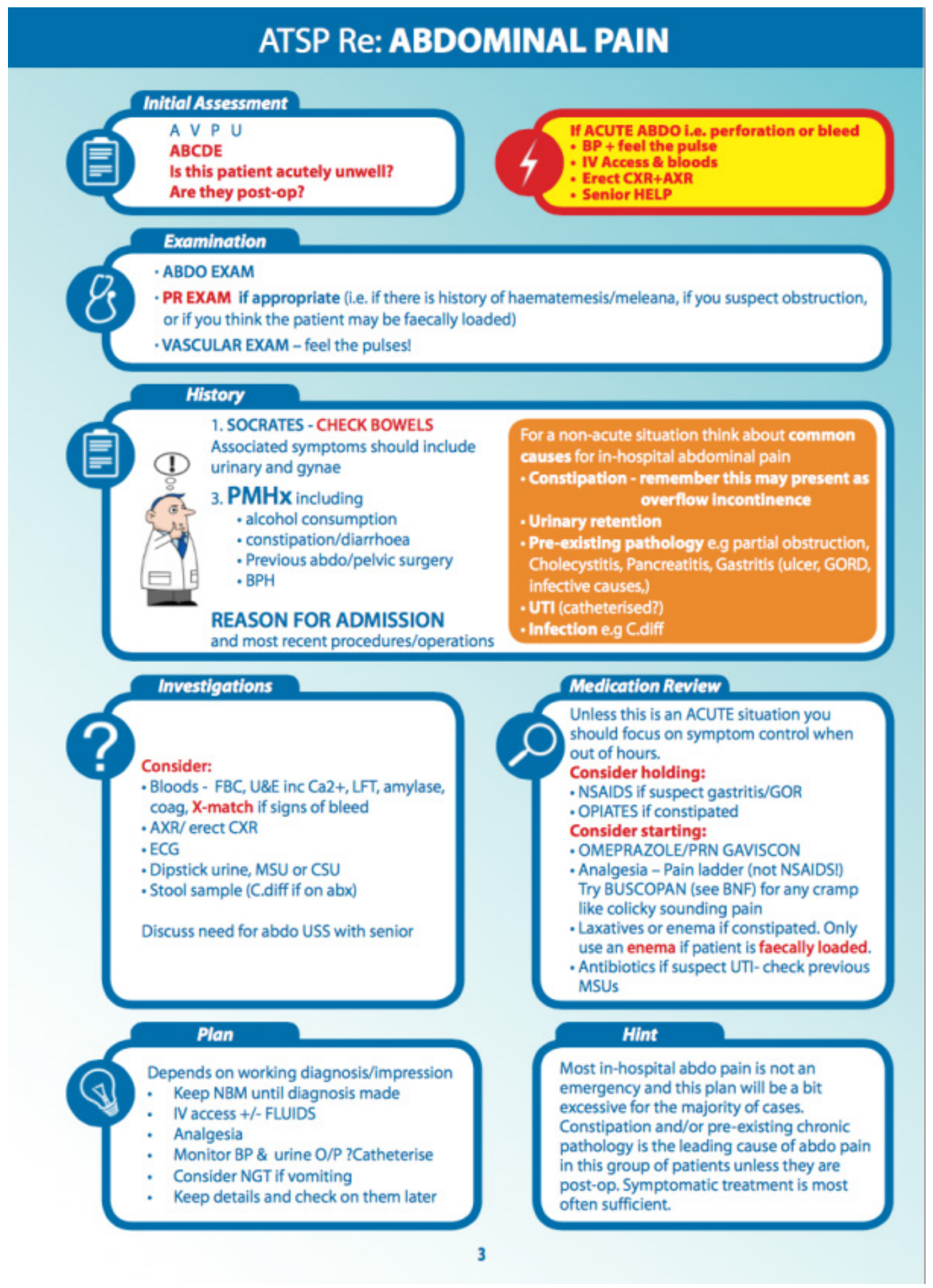

Figure 3. Example of ATSP clinical scenario in the booklet.

The booklet is now also available in an electronic format as a mobile-friendly Website available on tablets and phones (Android and iOS) to make it more accessible out of hours, as well as being linked to their online portfolio.

The programme has been well received year on year by the new FY1 doctors and informal, verbal 
feedback implied that the programme was very popular. For 2013, we decided to collect formal written feedback from the new FY1 doctors across all 12 foundation programmes in the school, in order to assess the impact that the programme had on their confidence and organisation during their first few on-call shifts.

\section{Method}

One month following the teaching sessions, the new FY1 doctors were asked to complete an online questionnaire. The aim was to assess how useful the new doctors found the course, how frequently they referred to the booklet, and how the ATSP programme influenced their management of acutely unwell patients during on-call shifts. A mixture of visual rating scales and free-text questions were included and qualitative and quantitative feedback was obtained. The thematic analysis was divided into five key categories: (a) patient safety; (b) organization; (c) time management; (d) confidence; and (e) communication. Ethical approval was gained prior to initiating the project and no conflicts of interest were raised throughout the process.

\section{Results}

\section{Questionnaire Results}

A total of 548 FY1 doctors attended the ATSP course at their respective trusts in 2013 within the North Western Foundation School. Among the 548 doctors, 26\% of them (141) responded with feedback. Fifty-eight percent of them used the ATSP booklets during their on-calls and 20\% used the ATSP e-booklet. The results showed that $88 \%$ of new FY1 doctors (125) felt more confident approaching patients on-call (see Table 1), half of them (45\%) referred to the booklet at least 1-3 times during an average on-call shift (see Table 2), 76\% (107) felt that they were a safe and structured clinician as a result of the ATSP course (see Table 3), 77\% (109) reported feeling more confident in answering their on-call bleeps (see Table 4), and 59\% (83) reported improvements in prioritisation of tasks (see Table 5).

Table 1

I Feel More Confident in Approaching a Hospital Inpatient On-call as a Result of the ATSP Teaching

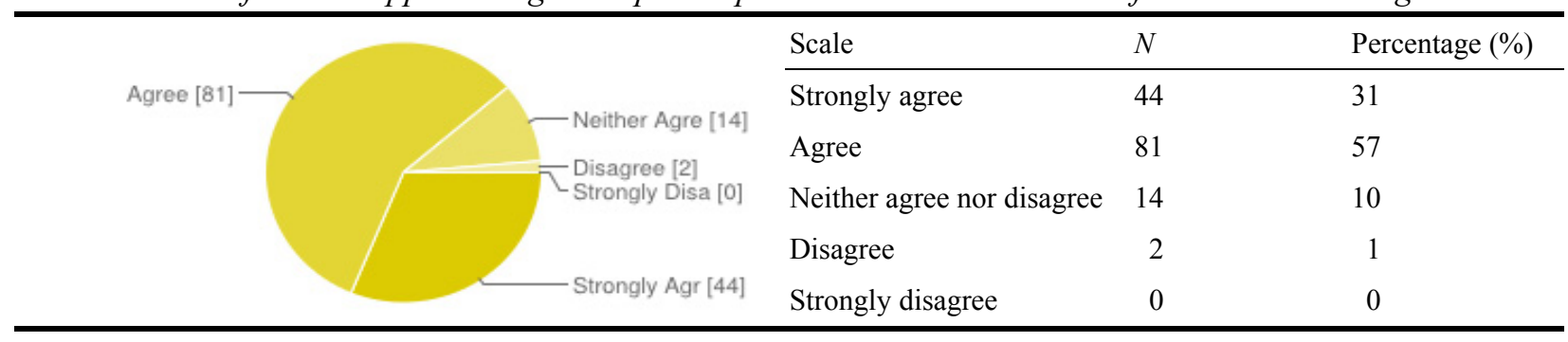

Table 2

How Many Times Did You Use Your ATSP Booklet During an Average On-call Shift?

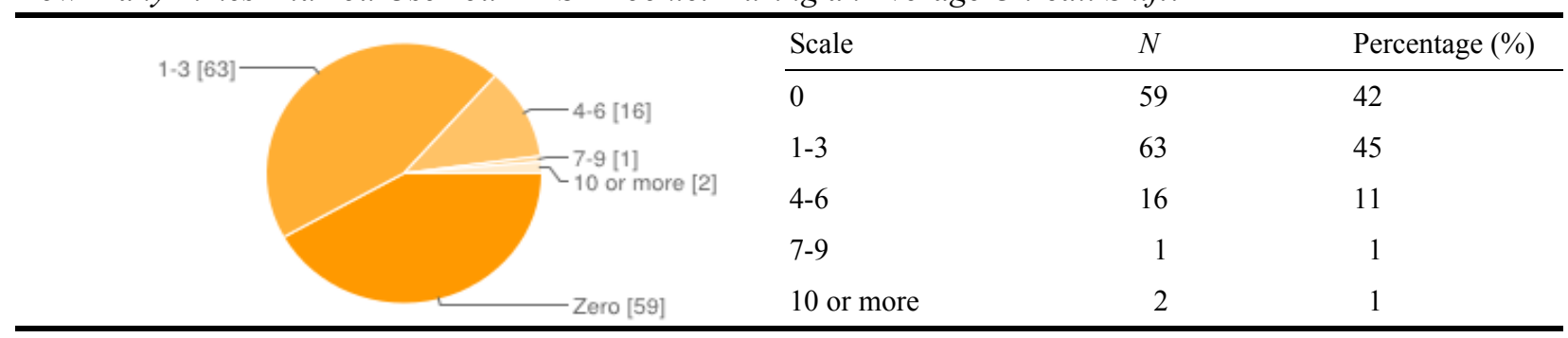


Table 3

As a Result of the ATSP Course, I Feel That My Practice as an On-call Clinician Is Safe and Structured

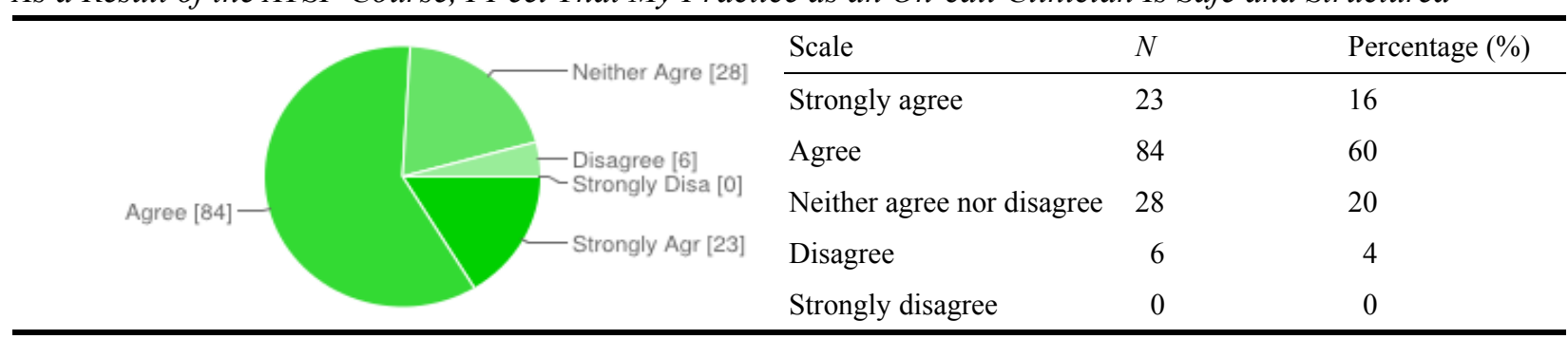

Table 4

As a Result of the ATSP Teaching, I Feel More Confident in Answering Bleeps On-call

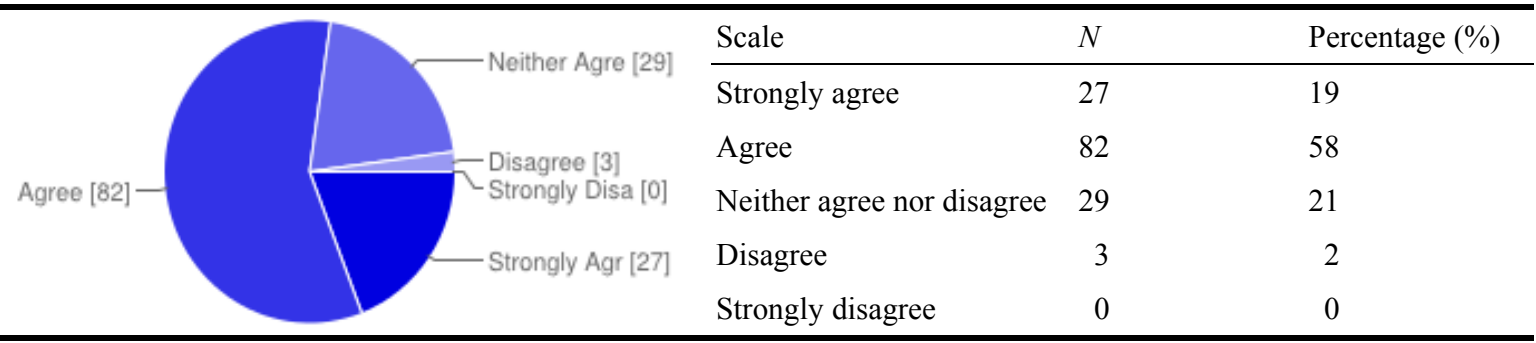

Table 5

I Feel That I Am Better at Prioritising Tasks On-call as a Result of the ATSP Teaching

\begin{tabular}{|c|c|c|c|}
\hline — Neither Agre [46] & Scale & $N$ & Percentage (\%) \\
\hline & Strongly agree & 12 & 9 \\
\hline [11] & Agree & 71 & 50 \\
\hline & Neither agree nor disagree & 46 & 33 \\
\hline & Disagree & 11 & 8 \\
\hline Agree $[71]$ & Strongly disagree & 1 & 1 \\
\hline
\end{tabular}

\section{Thematic Analysis}

Examples of direct quotes from the new FY1 doctors within the various categories that were assessed can be seen in Table 6.

Table 6

Qualitiative Feedback From New FY1 Doctors Who Attended the ATSP Course

\begin{tabular}{|l|l|}
\hline Thematic analysis categories & FY1 statements \\
\hline Patient safety & $\begin{array}{l}\text { 1. "When patients have had a high international normalized ratio (INR), I have used the booklet to } \\
\text { decide on management"; } \\
\text { 2. "It gave me a logical and safe set of instructions to follow and ensured I did not forget anything } \\
\text { crucial". }\end{array}$ \\
\hline Communication & $\begin{array}{l}\text { 1. "I write ATSP followed by ABCDE approach on the electronic patient record for every patient } \\
\text { as it gives a simple approach readable and clear to other doctors"; } \\
\text { 2. "It helps me structure my communication to the registrar when I need help". }\end{array}$ \\
\hline Organisation & $\begin{array}{l}\text { 1. "Allowed me to structure my thoughts before approaching patients and then whilst writing in } \\
\text { the notes after"; } \\
\text { 2. "I followed the structure in the book, now when I get called to see a patient I use this format } \\
\text { (redocumentation)". }\end{array}$ \\
\hline
\end{tabular}


(Table 6 to be continued)

\begin{tabular}{|l|l|}
\hline Time management & $\begin{array}{l}\text { 1. "I think my management would have been the same, but I might have spent longer thinking } \\
\text { about and looking up what to do"; } \\
\text { 2. "I now know what details I should ask for over the phone and what I could ask the caller to do } \\
\text { while I am on my way to see the patient, e.g., asking the nurse to do an electrocardiograph on the } \\
\text { patient with hyperkalaemia so that I could review it when I arrived on the ward". }\end{array}$ \\
\hline Confidence & $\begin{array}{l}\text { 1. "It served to reassure me that I was doing the right thing"; } \\
\text { 2. "I panicked, but ATSP reminded to follow the sensible structure I already know... i.e., history, } \\
\text { examination, investigations, plan". }\end{array}$ \\
\hline
\end{tabular}

\section{Discussion}

Patient safety surrounding the yearly August changeover period is a serious concern and has drawn much attention from the media, with pessimistic headlines warning the general public of "black Wednesday"; a day associated with compromised patient safety and increased clinical errors in hospitals (Jen, Bottle, Majeed, Bell, \& Aylin, 2009). Much of this negativity is aimed at newly qualified junior doctors, who only weeks before were final year medical students. It is important to emphasise that this negativity and fear is not a one-sided relationship. Many newly qualified junior doctors feel anxious when they begin their first day as a doctor; a doctor with real responsibilities, who has to face serious consequences if incorrect decisions are made. This transition is clearly a stressful and daunting experience.

Various online, interactive courses, e-books, and Apps are available to junior doctors to help them make this transition easier, in particular these resources focus on helping FY1 doctors convert their textbook-based medical knowledge into an applicable and clinically relevant format. However, many of these existing courses heavily depend upon the medical students or new doctors showing the initiative to find and attend them in their own time. ATSP is backed by Health Education North West and the teaching course is incorporated into the foundation induction programme at all of the hospital trusts in North West England, as an integral part of the foundation curriculum. As a result, all new North West foundation doctors automatically receive the teaching.

Junior doctors often feel overwhelmed by the degree of emotional, physical, and professional change they must undergo to become a working doctor. This can leave them feeling distant and dissociated from the various governing bodies, such as the General Medical Council who protect and support trainees. Peer-delivered training programmes allow new doctors to liaise with current foundation doctors, who can answer their queries and concerns and provide hints and tips about their new jobs. The key to the success of peer-delivered programmes is the insight that the teachers have into the challenges faced by their peers. In the case of the ATSP course, the teachers know first-hand the difficulties that new doctors face when they first qualify. This allows new doctors to make new friendships and develop mentors to approach for advice in both a clinical and non-clinical capacity (Connor, Bynoe, Redfern, Pokora, \& Clarke, 2000).

The timing of the course is a key consideration. The course is integrated into the foundation curriculum and delivered as an essential component of the induction period. This eliminates the burden on the new doctors to organise time for extra courses before they start work and ensures that the skills they acquire from the course are fresh and ready for utilisation on upcoming on-call shifts (Sukcharoen, Everson, \& Van Hamel, 2014).

The aim of the ATSP course is to address some of these concerns regarding patient safety and new doctor anxiety, by bridging this gap from medical students to calm, organised, and safe foundation doctors. The 
positive results demonstrate that the new doctors found ATSP helpful during this transition. The majority of those surveyed agreed that the ATSP course improved their confidence when answering bleeps, enabled them to practice in a more structured and safe manner, and aided their prioritisation of tasks.

Thematic analysis of the data was essential, as the feedback from the open space questions comprised of opinions from the new FY1 doctors and specific examples of scenarios where they felt that the ATSP session aided their clinical management. These qualitative data showed that the ATSP course successfully empowered new FY1 doctors to comfortably manage patients out of hours. They referred to the booklet for guidance on key points to be aware of when initiating management of acutely unwell patients, prior to calling for senior help. Overall, the doctors felt calmer when approaching patients out of hours and more confident to document their assessment and management plan clearly in the notes.

Peer-delivered teaching is relatively cost-effective to run. We found great interest from existing FY1 doctors to deliver the ATSP session at their respective trusts, likely because of the benefit they received from attending the course themselves. Furthermore, the foundation curriculum requires all junior doctors to demonstrate teaching within the workplace in order to successfully progress onto higher training. ATSP serves a dual function to new trainees and existing doctors as part of their continuing professional development.

ATSP does have some limitations. So far, the course has only been delivered to one school. However, in 2014, the programme was delivered to hospital trusts within the Mersey region for the first time. This expansion allowed the course to be delivered to a total of 812 new FY1 doctors. It is hoped that the ATSP course will continue to expand over future years, in order to benefit more new FY1 doctors across the United Kingdom.

The course also provided the doctors with a reference booklet, which was easily lost and potentially cumbersome to carry around. A mobile-friendly website has now been developed and will be introduced to the ATSP course for the first time in 2015. This application is linked to the Health Education North West foundation portfolio and can be carried around on smart phones and computerised tablets, making it even easier for new doctors to access the course material, whilst working on busy hospital wards.

\section{Conclusion}

ATSP is a peer-delivered teaching programme delivered to newly qualified FY1 doctors in the North Western Foundation School, prior to commencing their new jobs. The programme imparts key skills around answering bleeps, the initial management of unwell patients, and documentation in patient notes. The participants feel more confident and better equipped to initiate first-line assessment, investigation, and management of patients prior to obtaining senior help. The ATSP programme successfully bridges the gap between medical student and newly practicing doctor.

\section{References}

Brennan, N., Corrigan, O., Allard, J., Archer, J., Barnes, R., Bleakley, A., ... Regan, S. (2010). The transition from medical student to junior doctor: Todays experiences of tomorrow's doctors. Medical Education, 44(5), 448-458.

Connor, M. P., Bynoe, A. G., Redfern, N., Pokora, J., \& Clarke, J. (2000). Developing senior doctors as mentors: A form of continuing professional development. Report of an initiative to develop a network of senior doctors as mentors. Medical Education, 34(9), 747-753. 
Dornan, T., Boshuizen, H., King, N., \& Scherpbier, A. (2007). Experience-based learning: A model linking the processes and outcomes of medical students' workplace learning. Medical Education, 41(1), 84-91.

Glynn, L. G., MacFarlane, A., Kelly, M., Cantillon, P., \& Murphy, A. W. (2006). Helping each other to learn-A process evaluation of peer assisted learning. BMC Medical Education, 6(1), 18.

Jen, M. H., Bottle, A., Majeed, A., Bell, D., \& Aylin, P. (2009). Early in-hospital mortality following trainee doctors' first day at work. PLoS One, 4(9), e7103.

Sukcharoen, K., Everson, M., \& Van Hamel, C. (2014). A novel approach to junior doctor induction: A near-peer based curriculum developed and delivered by outgoing Foundation year doctors. BMJ Quality Improvement Report, 3(1). doi: 10.1136/bmjquality.u203556.w1603 